Théologiques

Théologiques

\title{
Humilité : l'éthique de la foi
}

\section{Howard S. Joseph}

Volume 8, numéro 2, automne 2000

Le pouvoir

URI : https://id.erudit.org/iderudit/005011ar

DOI : https://doi.org/10.7202/005011ar

Aller au sommaire du numéro

\section{Éditeur(s)}

Faculté de théologie de l'Université de Montréal

ISSN

1188-7109 (imprimé)

1492-1413 (numérique)

Découvrir la revue

\section{Citer cet article}

Joseph, H. S. (2000). Humilité : l'éthique de la foi. Théologiques, 8(2), 127-143. https://doi.org/10.7202/005011ar

\section{Résumé de l'article}

La catégorie de l'humilité théologique met en évidence la nature infinie del'Etre mystérieux de Dieu, la nature finie de notre compréhension et, parconséquent, la possibilité d'une pluralité d'approches légitimes du divin.Divers groupes religieux aperçoivent le divin grâce au sens cohérent qu'ilsdonnent aux événements et aux traditions dont ils héritent.

L'humilitéthéologique, cependant, oblige les croyants à se rappeler qu'il s'agit de leurvérité, et qu'elle constitue par définition seulement un aperçu partiel del'infini, suffisant néanmoins pour façonner et engager toute la vie d'unecommunauté. Ce point de vue peut fonder un pluralisme qui nous permet detenir en même temps notre croyance en la vérité de notre propre tradition etl'affirmation de la vérité de la foi des autres. d'utilisation que vous pouvez consulter en ligne.

https://apropos.erudit.org/fr/usagers/politique-dutilisation/ 


\section{Humilité : l'éthique de la foi}

Howard S. JOSEPH ${ }^{1}$

Dans la tradition juive, l'humilité est une qualité qui comporte toute une gamme des nuances possibles entre la modestie et l'estime de soi. Je veux expliquer ici pourquoi je crois que cette vertu et ce que j'appelle l'humilité théologique, devraient jouer un rôle important dans la vie religieuse. En effet, l'une des caractéristiques inquiétantes d'une bonne partie de la religiosité contemporaine est l'absence d'humilité. On s'en rend compte en constatant l'ampleur du fanatisme qui s'autolégitime ou en voyant comment des groupes religieux se retirent du monde pour se replier sur eux-mêmes.

Les personnes qui sont confrontées à la diversité des croyances et des styles de vie dans le monde moderne doivent parvenir à un compromis acceptable sur la question du particularisme et de l'universalisme. Le défi spirituel et intellectuel le plus difficile auquel nous avons à faire face a rapport au problème de la vérité que cette situation implique : si ma religion est vraie, absolument vraie, quel est alors le statut des autres religions et celui des croyances de leurs adeptes qui sont convaincus que leurs croyances sont vraies, absolument vraies?

Il nous arrive souvent d'ignorer ou de réprimer cette question, ou tout simplement d'adopter l'attitude de tolérance démocratique à laquelle on s'attend dans une société ouverte et libre. Cela peut constituer une solution satisfaisante dans plusieurs cas ; mais quand il s'agit de religion, c'est-à-dire des choix de Dieu et non les nôtres,

1. L'auteur est le rabbin de la synagogue Spanish Portuguese de Montréal, Québec, et professeur adjoint au Département de sciences religieuses de l'Université Concordia de Montréal. Une première version de cet essai a été présentée comme communication principale lors du Congrès international de la conférence des chrétiens et des Juifs, à Montréal, en 1998. 
l'obligation de prendre parti semble plus pressante. Les religions et leurs adeptes ont tendance à considérer les choses d'une manière absolue. Au mieux, on peut en venir à réaliser que la question que pose l'existence éventuelle d'une diversité de vérités religieuses, inscrites dans de multiples religions, relève du mystère de la foi. Mais on peut se demander s'il y a un cadre conceptuel susceptible de nous amener plus loin - au-delà du mystère et de la tolérance, jusqu'à l'affirmation religieuse des fois qui sont différentes de la nôtre?

Les Juifs ont habituellement considéré les autres religions comme des faussetés. Malgré cette vision globale des choses, ils ont cependant reconnu que les deux groupes religieux principaux parmi lesquels ils vivaient, les chrétiens et les musulmans, avaient un certain rapport avec la religion véritable dans la mesure où ils avaient emprunté et perpétué des enseignements bibliques au sujet de Dieu et du comportement humain ${ }^{2}$. Ce point de vue à été soutenu même dans des situations d'oppression et d'humiliation, même quand la légitimité du Judaïsme était niée sous prétexte que cette religion avait été supplantée par d'autres. Le rappel de ce contexte nous permettra de voir que cette attitude est plus remarquablement généreuse qu'il n'y paraît à première vue.

Ainsi, on reconnaissait que l'Islam et le Christianisme contribuaient à faire disparaître l'idolâtrie de la surface de la terre, de même que les débordements de violence et d'immoralité que la Bible associe à l'idolâtrie. Ils ont aussi diffusé l'idée du Dieu créateur qui donne la Torah et les commandements ; ils ont également préparé le monde à la venue véritable du messie, à une époque où les méprises concernant la doctrine juive auront été clarifiées. Entre-temps, on exprime souvent l'espoir que les croyants individuels de ces religions vivront en conformité avec les codes moraux et religieux qu'ils ont déduits de la Torah. On appelle parfois ce code les Sept commandements de Noé, c'est-à-dire les commandements que Noé aurait reçus après le déluge pour garantir que les humains ne s'abaisseraient plus au niveau antédiluvien de violence et d'immoralité. De même, on reconnaît l'exis-

2. On trouve de nombreux exemples de cette attitude. Voir Maïmonide, Code, Juges (Rois 11,4). (L'édition du Mossad Harav Kook contient le texte restauré avant censure). Voir Judah Halevi, Kuzari $(4,23)$. Pour un exemple souvent négligé, voir le commentaire de David Kimhi sur l'Aqédah (Gn 22). Je reviendrai sur ces textes plus loin. 
tence de ceux qui peuvent d'ores et déjà être comptés parmi les justes et les pieux des nations, les zadikei ou hasidei umot ha-olam.

De grands penseurs juifs tels que Maïmonide et d'autres ont élaboré ce point de vue au cours du Moyen Âge. On doit donc le prendre très au sérieux avant de passer à autre chose. Pour plusieurs de nos contemporains, il représente le meilleur traitement que les croyants d'autres religions peuvent espérer de la part du Judaïsme. Certains diraient qu'il n'y a plus rien à ajouter à propos des autres systèmes de croyances. Nous pouvons vivre dans la paix et la tolérance, en respectant les autres êtres humains qui sont des créatures de Dieu, même si nous n'avons aucune opinion à propos de la vérité de ce qu'ils croient.

Une telle position serait acceptable si nous avions une neutralité absolue par rapport à cette question. Même dans ce cas, ne resteraitil pas malgré tout des attitudes implicites vis-à-vis des autres religions? Ces attitudes seraient-elles vraiment neutres? Et si elles ne l'étaient pas, seraient-elles plutôt favorables ou défavorables à l'égard des autres religions?

À mon avis, il est impossible de maintenir une telle position ; cela peut même s'avérer dangereux. Il y a présentement, par exemple, un mouvement dans le sens du dialogue interreligieux et de l'examen critique des énoncés de sa propre tradition à l'égard des autres. Cette activité dialogale relève pour une bonne part de la prise de conscience que certaines significations implicites de nos discours peuvent prendre des formes particulièrement hargneuses. Des propos tenus dans une communauté, qui ne se voulaient que des mots, peuvent parfois susciter et justifier des gestes meurtriers qui dépassent de loin l'intention originelle de ceux qui les ont prononcés. Je fais évidemment référence à notre expérience de l'Holocauste, au $20^{\mathrm{e}}$ siècle, qui nous a montré comment des croyances et des attitudes sévères peuvent aller jusqu'à influencer notre comportement à l'égard d'autrui. Les gestes finissent par rattraper les paroles et, éventuellement, le processus de déshumanisation qui en résulte peut mener à la destruction.

Un telle prise de conscience est sous-jacente à une bonne partie des activités de dialogue. Il faut féliciter de nombreuses églises qui ont fait de courageux efforts pour se confronter à leurs propres traditions de mépris à l'égard des Juifs et du Judaïsme. Ils ont démontré qu'une telle entreprise est urgente et qu'il est impossible de laisser les choses aller à la dérive tout en espérant qu'elles aient une fin heureuse. 
Cela nous ramène à notre sujet, qui représente un défi majeur pour les Juifs comme pour les autres. Quel est le lien entre la voie que nous adoptons et les voies multiples de ceux au milieu desquels nous vivons?

Le point de vue médiéval semble donc poser plusieurs problèmes. Il porte un jugement sur les autres à partir de soi-même, ce qui est contraire au sage conseil donné dans l'Éthique des pères : "Ne juge pas ton prochain tant que tu n'es pas dans la même situation ". Il respecte les individus en tant qu'êtres humains, mais pas quand ils constituent un groupe qui célèbre ses croyances les plus précieuses. De plus, tout en reconnaissant, dans ces systèmes de croyances, des parcelles de vérité qui proviennent du nôtre, il ignore totalement la question de la diversité des croyances religieuses dans la communauté humaine ${ }^{3}$.

L'ouverture au pluralisme peut être un dérivé du commandement d'aimer les autres qui constitue la base de l'attitude éthique religieuse. Nous sommes mis au défi d'étendre cet amour d'autrui non seulement au voisin qui partage nos croyances et nos valeurs, mais aussi à l'étranger qui vit au milieu de nous. On peut supposer que l'étrangeté de l'étranger ne se limite pas au fait d'être né ailleurs, mais qu'elle inclut aussi des croyances et des valeurs qui nous paraissent étranges. L'ouverture à l'autre peut paraître très difficile ; c'est néanmoins l'un des buts les plus nobles auxquels on puisse tendre. Quiconque réussirait à surmonter sa peur de l'altérité de l'autre et à lui substituer le respect, l'attention et l'amour réussirait quelque chose de grand et donnerait un exemple de l'amour à son meilleur.

Toutefois une telle approche ne répond pas vraiment à la question de la légitimité des croyances de l'autre. On peut soutenir que cette légitimité serait affirmée sur la base du même amour d'autrui, en dépit du caractère absolu de ma foi. Dans cette optique, les contradictions apparentes sont résolues dans le mystère du Dieu infini. On a cependant affaire ici encore à une forme de tolérance et non à une affirmation de la vérité de ce qui est le plus précieux aux yeux de l'autre. Poussée à la limite, une telle attitude conduit à afficher ses propres croyances avec arrogance et à manifester de la condescendance vis-à-

3. Mehahem Ha-Meiri constitue une exception à cet égard, car il parle de manière positive de tous les peuples qui sont liés par des traditions religieuses et morales. 
vis des autres, ce qui va certainement à l'encontre de l'idéal d'humilité qui, avec l'amour, est un des traits caractéristiques de la piété. Cette arrogance repose sur la certitude que mes propres croyances sont clairement exposées, alors que celles des autres demeurent nébuleuses et mystérieuses, voire menaçantes pour les miennes. Mes croyances occupent tout le territoire de la légitimité, ne laissant aucun espace théologique pour celles d'autrui. Ainsi, paradoxalement, même si la rencontre de l'étranger peut accroître notre capacité d'aimer, elle peut réduire notre humilité. Ceci peut nous amener à édifier un mur protecteur autour de notre propre foi pour éviter d'avoir à nous confronter en toute sincérité à l'apport d'autres points de vue ou d'autres idées. C'est qu'en réalité, le défi que l'autre représente pour nous ne se limite pas au plan éthique, mais il a aussi une dimension réflexive, intellectuelle, et, pour le fidèle, théologique.

C'est pourquoi la recherche d'un cadre théorique pour le pluralisme religieux est une nécessité. La catégorie de l'humilité théologique en face du Dieu infini et mystérieux est, je pense, un pas dans la bonne voie. Elle met l'accent sur la nature infinie de l'Être de Dieu, sur la nature finie de notre compréhension, et, par conséquent, sur la possibilité d'une pluralité d'approches légitimes du divin.

Le défi que pose l'expérience moderne de la sécularité est un incitatif pour la recherche d'une base religieuse au pluralisme. La modernité a vu dans la religion une source d'intolérance et d'exclusion, car, dans la société prémoderne, la religion déterminait l'appartenance à une société ou l'exclusion de celle-ci.

Au siècle des Lumières, les penseurs ont fondé la société sur une base nouvelle : les droits naturels et individuels de chacun. La religion ne serait désormais qu'une affaire privée, sans intérêt pour la structure politique en tant que telle.

Les nouveaux États fondés sur ces principes démocratiques particulièrement dans le Nouveau Monde — ont attiré des gens venus de partout, qui fuyaient l'intolérance et la persécution religieuse. Mais, dans cet environnement plus ouvert, plusieurs ont perdu la foi tandis qu'ils s'intégraient dans la société nouvelle et contribuaient avec d'autres à la création d'une culture commune, séculière, partagée par tous et n'appartenant en propre à personne. Ceux qui tentaient de conserver un engagement intense à l'égard de leur foi et de leur culture d'origine ont eu à apprendre à tolérer — souvent malgré eux — la 
présence de plusieurs autres systèmes de croyances. C'est ce qui a donné son élan au pluralisme.

Le pluralisme est donc, en définitive, un produit du siècle des Lumières et de son insistance sur la Raison comme source des valeurs. La Raison a créé l'État moderne et la société ouverte qui en résulte. Une démocratie est un processus en développement qui requiert une vigilance constance et un discours rationnel pour maintenir la liberté de ses membres.

Ceux qui ont mis sur pied la société moderne ont peut-être espéré qu'avec le temps, les différences significatives entre les citoyens allaient diminuer ou disparaitre ; une culture commune en surgirait, qui lierait les citoyens entre eux ${ }^{4}$. Cette culture pourrait même comporter des éléments de spiritualité et ce qu'on appelle souvent une " religion civile ». En dépit de ces attentes, on constate la persistance remarquable de plusieurs religions traditionnelles. Elles se sont refusées à disparaître et elles continuent d'attirer des croyants engagés et à prendre en charge la vie spirituelle de millions de personnes. Cette persistance, tout comme la revitalisation actuelle de la foi rend urgente la nécessité d'élaborer un pluralisme religieux fondé sur des principes adéquats. Dans un système basé sur la Raison et le discours rationnel, une approche religieuse rationnelle est plus sécurisante et plus attrayante. Les religions n'ont pas à être perçues comme une source de conflit et d'intolérance, et la persistance et la revitalisation de la religion n'est pas nécessairement une menace pour la société démocratique et ouverte. Les religions peuvent inclure un certain degré d'ouverture dans leur propre autocompréhension. La question du pluralisme peut ainsi être considérée comme un autre chapitre de l'histoire continue des relations entre la raison et la révélation, entre la foi et la connaissance.

4. En 1818, Mordecai M. Noah diffusa des copies d'un discours prononcé lors de la dédicace de la deuxième synagogue de Mill Street de la Shearith Israel de New York. En réponse, James Madison écrivait: « Ayant toujours considéré que la liberté des opinions religieuses et du culte appartiennent à toute secte, et que la jouissance paisible de ce privilège est le meilleur moyen humain pour amener tout le monde soit à la même façon de penser, soit à une charité mutuelle qui en est le seul substitut acceptable.... » Dans David Desola PoOL, An Old Faith in the New World, New York, 1955, p.452. 
Les croyants doivent humblement reconnaître avoir péché en persécutant, en supprimant et en dénigrant les autres religions ${ }^{5}$. Sans le défi que pose l'expérience de la modernité, nous ne serions peut-être pas parvenus à cette occasion nous élever au-dessus de cette faillite historique. La rencontre de l'intégrité des autres au sein d'une société libre nous a incités à renouveler et à rafraîchir notre autocompréhension. Nous pouvons vivre à la fois avec humilité et avec amour.

Ce qui est en cause ici, en définitive, c'est le défi de la raison face à la révélation. Pour les prémodernes, cette question demeurait sur le plan intellectuel — théologique. C'est aujourd'hui au cœur de l'expérience sociale moderne qu'elle se pose ; cela est encore plus troublant et beaucoup se refusent à l'aborder. Pour quelques-uns, cependant, il s'agit là d'une source constante d'enrichissement et de renouveau sur la voie d'une meilleure compréhension du mystère de Dieu et de la majesté complexe de la création.

Ce problème comporte un ensemble de questions étroitement imbriquées l'une dans l'autre. Lorsqu'on se demande si une religion est vraie ou contient une certaine vérité, nous prenons pour acquis que nous savons ce que nous voulons dire en utilisant le mot vérité lorsqu'il s'agit de religion, y compris la nôtre. Mais est-ce bien dans la nature de la religion de parler de certitude et de "vérité» de la même manière que dans les affaires humaines courantes? Que voulons-nous donc dire par les mots foi, confiance, croyance?

L'humilité est une caractéristique qui comprend à la fois la modestie et l'estime de soi, mais qui évolue plutôt dans le segment de la modestie à l'intérieur d'un continuum qui se déploie entre ces deux qualités. ${ }^{6}$ La personne humble évite les deux extrêmes, ne faisant montre ni d'arrogance ni d'un effacement de soi indigne d'une créature faite à l'image de Dieu, et avec laquelle son créateur aimant peut entrer en rapports pour l'interpeller et lui commander. Ils vivent dans

5. En réponse à la même allocution de Noah, Thomas Jefferson écrivait: "Votre secte, par tout ce qu'elle a souffert, a donné une preuve remarquable de l'esprit universel d'intolérance inhérent à toute secte, qui est dénoncé par tous lorsqu'ils sont en position de faiblesse et qui est pratiqué par tous lorsqu'ils ont le pouvoir.... »Ibid.

6. Voir MAÏMONIDE, Commentaire de la Mishnah, Introduction au Traité Abot (L'Éthique des Pères), connu sous le nom des Huit chapitres, ch. 4. 
l'humilité, mais avec assurance, confiants qu'il s'agit là d'une disposition essentielle envers le monde qui les entoure.

L'humilité n'est pas importante seulement dans le domaine des relations interpersonnelles. Il s'agit également d'un qualité théologique, d'une catégorie de pensée qui décrit des tendances qui sont déjà présentes dans la réflexion théologique. Lorsqu'elle est définie ainsi, elle devient un concept très utile, qui peut nous servir de guide lorsque nous abordons des questions religieuses ${ }^{7}$.

L'humilité théologique trouve sa forme d'expression la plus claire dans l'épisode de la deuxième rencontre de Moïse et de Dieu sur le mont Sinaï, rapporté en Exode 32-34. Moïse s'efforce de profiter de ce moment privilégié pour acquérir la meilleure compréhension de Dieu possible. Il demande avec insistance à Dieu de contempler totalement sa Gloire ou sa Présence. Il se fait répondre que cela est impossible : aucun être humain ne peut atteindre ce but. Même si les humains peuvent voir la Bonté de Dieu, «tu ne pourras pas me contempler de face, car les humains ne peuvent me voir et rester en vie ». Dieu lui dit finalement : «tu me verras de dos, mais tu ne me verras pas de face. »

Ce texte nous a souvent poussés à être des théologiens hésitants ou modestes. S'il n'y a aucune possibilité de venir à bout de notre sujet, alors pourquoi nous en préoccuper?

Mais la leçon véritable est beaucoup plus profonde. Le texte nous enseigne que ce moment spirituel privilégié n'aboutit pas à une connaissance absolue. La rencontre avec l'Absolu est comblante et convaincante, mais ne mène pas à une description rigoureusement exacte de la Divinité. On voit Dieu de dos, mais pas de face. Notre tentative de connaître Dieu doit demeurer inachevée. Malgré tout, le croyant inspiré continue d'être un fidèle après une telle rencontre. Ce qu'il a vu lui suffit.

7. Alors que je réfléchissais à cette idée, je me suis rendu compte que l'expression "modestie théologique » a été utilisée, et ce, en rapport avec quelques-uns des textes dont je vais traiter, mais pas tous. Henry Seigman a utilisé l'expression "modestie théologique " pour parler de notre incapacité à parvenir à la précision en théologie. Voir son essai " Ten Years of CatholicJewish Relations: A Reassessment ", dans Fifteen Years of Catholic-Jewish Dialogue 1970-1985, Roma, Libreria Editrice Vaticana, 1988, p. 31. 
Ceci ressort clairement du commentaire de Maïmonide sur l'expérience de Moïse ${ }^{8}$. Il nous rappelle que toutes les descriptions de Dieu ne sont que des métaphores ou des analogies qui ne doivent pas être comprises littéralement. "La Vérité de la Présence de Dieu ne peut être ni comprise, ni perçue, ni scrutée. Qu'est-ce donc que Moïse a demandé au Dieu Saint?... Il a demandé à connaître la réalité de l'existence de Dieu de telle sorte que sa connaissance soit semblable à celle d'une personne dont on a vu la face et qu'on connaît maintenant comme un être unique. »Cela était impossible. Mais «Dieu lui révéla quelque chose qu'aucun être humain n'avait connu avant lui : il lui a fait percevoir quelque chose de l'existence de Dieu qui suffirait à graver dans son esprit le caractère unique de Dieu par rapport à tout être. » Maïmonide explique donc la métaphore du texte biblique. C'est semblable à ce qui se passe quand on ne voit que le dos d'une personne, mais que cela suffit à reconnaître que c'est bien de cette personne qu'il s'agit.

Maïmonide ne dit pas quelle est cette chose particulière qui est révélée à Moïse et qui lui permettrait de reconnaître Dieu de dos, pour ainsi dire. Mais, dans le texte, Dieu parle de "passer devant toi en te montrant toute ma bonté et en proclamant mon nom "Le Seigneur" devant toi : j'aurai pitié de qui j'aurai pitié et j'aurai compassion de qui je veux avoir compassion. »

Selon la tradition juive, ce jour est devenu le Yom Kippour. C'est le jour où la faute du veau d'or a été pardonnée. C'est le jour où le message de l'amour gratuit de Dieu a été proclamé haut et fort.Au moment du Yom Kippour, comme à d'autres occasions où ce moment est évoqué, le nom de Moïse n'apparaît pas dans le texte de la prière. Au lieu ce cela, on le désigne comme "l'homme humble ». Cela nous rappelle que, de toutes les qualités que Moïse possédait sans doute, c'est pour son humilité qu'il est le plus estimé.

Dans le livre des Nombres, aux chapitres 11-12, on présente Moïse comme "un homme humble, plus que toute personne sur terre ». Cela nous renvoie à la perception que Moïse avait de son propre honneur et de sa responsabilité. Il ne recule pas devant la possibi-

8. Code, I (Mada), Yesodei Hatorah, Ch.1/9-10. Le rabbin Dr. Emanuel Rackman, mon maître, est celui qui m'a signalé ce texte en premier. L'application que j'en propose est la mienne. Le rabbin Rackman a par la suite donné son aval à mon interprétation. 
lité de partager l'esprit divin avec les autres pour que le peuple soit gouverné correctement. "Si seulement tous les Israélites devenaient prophètes, si seulement le Seigneur répandait son esprit sur eux! » Il choisit d'ignorer les calomnies énoncées par Miriam et Aaron à son sujet, assuré d'avoir agi en conformité avec sa mission. Ainsi, au Yom Kippour et à d'autres occasions, lorsque nous souhaitons nous rappeler le message de l'amour et de la bonté divine, c'est Moïse l'anav, l'humble, que nous évoquons. C'est cette humilité qui a rendu possible la révélation qui constitue maintenant le cœur de l'espérance du pardon pour ses disciples reconnaissants.

Le Moïse de la maturité est passablement différent de celui que la Bible nous présente au début du livre de l'Exode. Jeune prince d'Égypte, il décide de quitter le palais et d'aller vers ses frères. Plein d'un enthousiasme juvénile peut-être teinté d'arrogance, il frappe un contremaître égyptien qui battait un esclave hébreu. Pensait-il libérer Israël d'un seul coup? Contraint de quitter l'Égypte et de s'exiler en Madian pendant plusieurs années, il devient complètement impuissant pour secourir son peuple. Il a appris l'humilité de manière forte.

Au buisson ardent, Moïse continue d'apprendre l'humilité. Ici, il s'efface beaucoup trop, ne cessait de s'objecter contre son choix comme messager de salut. Il est passé d'un excès de confiance à un abaissement extrême. Il élabore une série d'arguments contre le projet de salut, de sa propre inefficacité à l'impossibilité d'obtenir l'accord du Pharaon et de réveiller un nation démoralisée qui languit en captivité.

Moïse doit être formé pour assumer son rôle de leader. L'humilité, la patience et la reconnaissance que les situations humaines sont complexes et que des reculs - tel que l'épisode du veau d'or - sont toujours possibles, sont toutes des qualités requises de quelqu'un qui doit conduire un peuple vers son salut. Lorsqu'il retourne à la montagne, après l'Exode, la révélation du Sinaï et l'épisode du veau d'or, - il apprend les limites de la réussite humaine : les êtres humains ne peuvent Me voir et vivre. C'est un Moïse humble qui accepte de ne pas voir Dieu de face. Personne ne peut apercevoir ou comprendre la totalité de la gloire de Dieu. On doit se contenter de le voir de dos.

Ces textes suggèrent quelque chose des limites de la connaissance religieuse : notre vision et les mots que nous utilisons pour décrire une rencontre de Dieu sont au mieux incomplets, finis, et partiels. Le Dieu 
mystérieux ne peut jamais être saisi totalement. Le Dieu infini peut être contemplé à partir d'une infinité de points de vue, chacun pouvant s'avérer ultimement aussi adéquat que les autres. Par définition, un point de vue ne peut être infini ; il ne peut englober tous les points de vue possibles qui constitueraient la vérité entière sur l'existence de Dieu.

Une telle position peut paraître relativiste. Mais il y a une grande différence entre l'affirmation que toutes les religions sont essentiellement la même chose et l'affirmation que plusieurs religions peuvent être valables ou vraies. La première position implique qu'il n'y aurait aucune différence significative entre les religions, alors que la seconde soutient précisément le contraire. Ces différences peuvent concerner les points de vue et les vérités théologiques, mais elles peuvent aussi être en relation avec les événements et expériences fondatrices sur lesquelles telle tradition est basée, et avec la communauté historique concrète qui proclame telle foi particulière. Pour chaque communauté, ses événements fondateurs et leur articulation en une religiosité vivante au cours des âges est quelque chose de sacré. Il y a donc beaucoup de différences entre les religions : des événements et leur interprétation, des conditions et expériences historiques, une littérature, un langage et une culture sacrée. Chacun de ces points de vue peut être une authentique perception du divin, irréductible aux autres. C'est l'une des innombrables manières dont le Dieu mystérieux et infini peut être perçu.

Le point de vue que je soutiens ici prend au sérieux les traits particuliers de chaque tradition. En ce sens, il évite le danger du nihilisme qui nierait la valeur de toutes les croyances et de toutes les morales sous prétexte qu'on ne peut élaborer aucun critère ultime et absolu. Ce point de vue s'apparente aussi à plusieurs courants philosophiques modernes qui sont arrivés à la conclusion que toute connaissance est relative, c'est-à-dire qu'elle dépend du consensus des individus qui l'acceptent et en vivent. Cela est vrai même de la connaissance scientifique. Elle aussi, en effet, est basée sur le consensus de la communauté scientifique. Ce qu'on considère comme étant la science change constamment à mesure que de nouvelles données sont découvertes et que de nouvelles théories sont proposées. Entre-temps, nous construisons des ponts et voyageons dans des avions comme si les principes sur lesquels se base leur construction étaient absolument sans risque. 
Avec la modernité, nous avons assisté à une véritable explosion de la connaissance qui nous est accessible. Ce matériel est organisé en de multiples catégories, chacune reflétant une manière d'aborder la réalité. Ainsi une donnée quelconque peut être analysée par la physique, la chimie et la biologie ; l'histoire, la sociologie et la psychologie. La religion peut aussi avoir quelque chose à dire à ce propos. L'esthétique peut aussi apporter un point de vue. La résultante est une pluralité de formes du savoir et de la connaissance : un pluralisme épistémologique.

De la même manière, le consensus à propos de la connaissance religieuse sur laquelle repose une communauté de foi particulière est un fondement sérieux et concret. La vérité devient donc le sens cohérent qu'une communauté donne aux événements et aux traditions dont elle hérite et à la manière dont cela contribue à l'organisation de la vie collective. Elle est supportée par l'autorité de ses chefs et est reconnue par ses fidèles. À travers cette vérité, ils espèrent apercevoir le divin et assumer dans leur vie personnelle et communautaire les responsabilités qui découlent de cette vision. L'humilité théologique, cependant, leur demande de se rappeler qu'il s'agit de leur vérité, et qu'elle constitue par définition seulement un aperçu partiel de l'infini. Ce qui est merveilleux, dans cette perspective, est que cet aperçu partiel est suffisant pour façonner et engager toute la vie d'une communauté.

Un tel point de vue, par conséquent, peut être la base d'un pluralisme sans relativisme. Ou, si l'on préfère, il peut être la base d'un nouveau relativisme ${ }^{9}$ qui associe la vérité à la perspective individuelle à partir de laquelle elle est formulée. Cela nous permet de tenir en même temps aux deux termes de notre problème - le particulier et l'universel, notre croyance en la vérité de notre propre tradition et l'affirmation de la vérité de la foi des autres.

Le scepticisme moderne caractéristique des débats en philosophie de la connaissance, ou l'épistémologie, et donc, le déplacement vers l'humilité théologique, se reflète dans la pensée juive récente. Le rab-

9. On pourrait parler de «relativisme objectif ». Ma réflexion sur ces questions d'épistémologie et de métaphysique a été largement influencée par les écrits de Justus Buchler, entre autres dans son livre Nature and Judgment and The Metaphysics of Natural Complexes. Jerome Eckstein m'a initié à ces travaux. En outre, mes lectures en anthropologie culturelle ont contribué à l'élaboration de ce point de vue. 
bin Abraham Isaac Kook, tout en reconnaissant que ce scepticisme provenait de la pensée kantienne, ne croyait pas qu'il s'agissait d'une idée nouvelle pour le Judaïsme. Dans une lettre rédigée en 1907, il écrivait :

Même le " passage à Kant » ne réussit pas à contenir la plus petite parcelle de la force d'Israël. En réalité, nous avons toujours su que tous les jugements humains sont subjectifs et relatifs et que nous n'avions pas besoin de Kant pour nous révéler ce secret... ${ }^{10}$

Le rabbin Kook écrivait également :

Il est impossible pour un être humain de connaître l'essence d'une chose, même de sa propre personne, et certainement celle d'autrui, qu'il s'agisse d'un individu ou d'un peuple. Nous gravitons autour du centre du savoir, affairés en estimations et en évaluations... et nous essayons de parler d'une personnalité unique et d'une âme particulière. Il nous faut admettre que la connaissance que nous en avons ne tient qu'à un fil et que le jugement appartient à Dieu ${ }^{11}$.

Partant de là, le rabbin Kook concluait que :

Par rapport à la Vérité divine supérieure, il n'y a pas de différence entre un système donné de croyances (ha-emunah ha-mezuyeret) et le scepticisme. Ni l'un ni l'autre ne correspond à la vérité. Toutefois la croyance est plus près de la vérité, tandis que le scepticisme est plus près de l'erreur... ${ }^{12}$

Le rabbin Joseph B. Soloveitchik a abordé ce sujet dans son essai de 1944, intitulé The Halakhic Mind ${ }^{13}$. Explorant la valeur de la philosophie et de la connaissance religieuse comme formes de connaissances, il conclut que le pluralisme épistémologique conduit au pluralisme métaphysique. Une fois établi de manière adéquate que nous vivons dans un univers aussi bien que dans une société pluraliste, la question abordée dans cet essai peut être soulevée : quelle est la relation entre notre connaissance et d'autres formes de connaissance?

10. Voir Mossad Harav KooK, Iggerot Reiyah, Jerusalem, 1985, p. 47. Je remercie le Dr. Tamar Ross pour cette référence aux travaux du rabbin Kook et celles qui suivent.

11. Mossad Harav Kook, Orot Hakodesh, Volume 3, Jérusalem, 1985, p. 119.

12. Mossad Harav KooK, Orot Ha-Emunah, Jerusalem, 1985, pp. 23-24. 13. Joseph B. Soloveitchik, The Halakhic Mind, Seth Press, New YorkLondon, 1986. 
La connaissance religieuse prétend être une connaissance absolue de l'Absolu. Mais si toute connaissance est en rapport avec un point de vue à partir duquel cette connaissance est acquise, une méthode par laquelle ce savoir est constitué, comment alors peut-on soutenir une quelconque prétention à l'absolu? La catégorie de l'humilité théologique permet de décrire et de définir cette réalité à laquelle la pensée religieuse moderne est sensible.

Peut-on dégager de l'analyse précédente des critères qui permettent à une tradition croyante d'en évaluer une autre? Y a-t-il des attentes légitimes que des communautés de foi peuvent entretenir l'une à l'égard de l'autre? Avec toutes les réticences et l'hésitation que suggère l'esprit d'humilité théologique, je propose ici quelques implications de cette réflexion qui peuvent être à la fois éprouvantes et interpellantes.

1. Un premier critère permettant d'évaluer la vérité d'un système religieux pourrait être l'humilité théologique elle-même, la reconnaissance autocritique de la nature limitée de toute compréhension humaine du divin. Ainsi, une voie spirituelle ou religieuse quelconque n'est qu'une voie, pas davantage. Toute prétention à être autre chose serait infidèle à l'expérience religieuse fondatrice.

Les croyants peuvent-ils parvenir à une telle reconnaissance? Doivent-ils affirmer la supériorité de leur point de vue sur celui des autres? Doivent-ils prétendre que leurs croyances ont une valeur universelle et tenter de les imposer aux autres? Ne leur suffit-il pas de se réjouir, de célébrer et de témoigner de leur propre système de croyances? Doivent-ils avoir la conviction que, pour que leur système soit vrai, il doit l'être pour tous et non seulement pour eux-mêmes?

2. Un autre repère pourrait être la leçon que Moïse a apprise lors du premier Yom Kippour. Personne ne peut saisir les voies de Dieu et prévoir la manière dont Dieu se révélera, car "J'aurai pitié de qui j'aurai pitié et j'aurai compassion de qui je veux avoir compassion. » Lorsque la Présence miséricordieuse de Dieu se manifestera, nous la reconnaîtrons comme telle. En d'autres termes, quand nous (démontrons) nous voyons de la pitié et de la compassion, nous rencontrons la divine Présence, même si cela se passe en dehors de notre propre communauté.

Je rappelle que je suis très hésitant lorsque je formule ces suggestions. Je les trouve éprouvantes, mais elles me réconfortent également, 
parce qu'elles nous défient de renoncer à une critique autojustificatrice des autres communautés et à nous examiner nous-mêmes sur notre propre humilité et notre capacité de reconnaître les manifestations de la grâce chez les autres.

3. La notion d'élection doit aussi être réexaminée. Y a-t-il place pour un tel concept dans la perspective de l'humilité théologique? Mon sentiment personnel à cet égard est un oui catégorique.

J'ai fait allusion plus haut à quelques traditions juives médiévales qui abordent la question des autres religions. Chacune reconnaissait dans ces religions autres un but divin pour lequel elles avaient été choisies. Maïmonide disait ceci tant du Christianisme que de l'Islam :

Les humains, toutefois, ne peuvent sonder les pensées (ou les projets) du Créateur, dont les voies et les pensées ne sont pas comme les nôtres. Toute l'affaire de Jésus de Nazareth et de l'Ismaélite (Mahomet) qui l'a suivi consiste à aplanir la voie pour le Roi Messie et à préparer l'univers entier à servir Dieu ensemble...14

Ainsi, même les penseurs juifs qui considèrent l'élection d'Israël comme un principe théologique fondamental peuvent imaginer en même temps un choix délibéré des autres. Nous ne comprenons jamais totalement le drame divin qui est à l'œuvre dans l'humanité. Nous ne pouvons sonder le mystère des autres religions par les critères qui nous sont propres. Chacune peut conserver sa conviction d'une élection particulière, mais elle doit laisser de la place pour les autres également.

La sensibilité culturelle de la fin du $20^{\mathrm{e}}$ siècle a changé à travers le monde. Il est à nouveau légitime de se distinguer et d'assumer sa différence ethnique ou religieuse. La religion redevient "à la mode ". Alors que nous sommes tentés d'accueillir favorablement ce changement, de sérieuses questions doivent être soulevées. La foi religieuse

14. Code, Juges (Rois 11,4). Les autres sources chez Halevi and David Kimhi notées précédemment expriment un point de vue similaire.

La conclusion de Maïmonide ici, "à servir Dieu ensemble " demande étude. Est-ce qu'il anticipe qu'il n'y aurait plus qu'une seule religion à la fin des temps, alors que tous seraient convertis au Judaïsme?

Plus récemment, le rabbin Naphtali Zvi Yehuda Berlin (1817-93), connu par l'acrostiche de son nom, Neziv, s'est attardé à cette question générale dans 
en ré-émergence répétera-t-elle les erreurs du passé, recréant un climat triomphaliste, arrogant et intolérant ? Ou sera-t-elle humble, purifiée par sa faiblesse au sein des sociétés séculières et par sa souffrance sous la répression des régimes totalitaires? Aura-t-elle appris quelque chose de valable de l'expérience pluraliste moderne ou tentera-t-elle de nous faire retourner à une manière de vivre prémoderne, dans des sociétés fondées sur l'homogénéité religieuse, l'exclusion et la ségrégation ? Ces questions devraient nous faire prendre conscience de l'urgence de reconnaître, à la fois la vérité et la valeur de "l'humilité théologique » comme concept à développer pour notre temps.

L'humilité théologique génère d'autres formes d'humilité également. Celui qui est vraiment humble évite l'arrogance et laisse de la place pour d'autres points de vue; il apprend des autres parce qu'il sait qu'il ne possède pas la vérité tout entière ; il laisse aussi un espace ouvert dans lequel la majesté mystérieuse de Dieu peut s'exprimer de manière toujours nouvelle et inattendue. Celui qui est vraiment humble a suffisamment d'estime de soi pour prendre la vie humaine au

Shear Yisrael (Le reste d'Israël ). Il soutient que puisqu'Abraham a été chargé de devenir " un père pour une multitude de nations, " le plan de Dieu n'est pas de faire disparaître les autres nations et de les fusionner à Israël. Neziv pense plutôt que le but de l'alliance avec Israël est de provoquer une élévation de la vie religieuse ou des alliances des autres nations. "Votre devoir est maintenant d'enseigner à toutes les nations la reconnaissance de Dieu et non de les convertir comme on l'a fait jusqu'à maintenant. L'intention de Dieu, depuis les origines, a été de maintenir l'intégrité de toutes les nations et non de les incorporer une à une à Israël. C'est aussi le désir de Dieu que tous les peuples en viennent à le reconnaître et à l'adorer, lui seul, et que l'idolâtrie cesse. Par conséquent, Abraham a reçu l'ordre de devenir le père de nombreuses nations, même sans leur conversion. "

Bien qu'il ne fasse pas référence à l'affirmation de Maïmonide ici, il la mentionne ailleurs. Voir le commentaire du Cantique des Cantiques Metiv Shir $(7,1)$. Il pourrait avoir supposé que Maïmonide aurait été d'accord avec lui.

Cet essai, Shear Yisrael, a été publié avec Metiv Shir. L'ouvrage combiné qui en résulte a pour titre Rinna Shel Torah, Le cantique de la Torah. Toutes les éditions hébraïques ont gardé les deux ensemble, selon le vœu de Neziv. Une traduction anglaise de Metiv Shir a été publiée récemment sous le titre Rinna Shel Torah mais sans Shear Yisrael. J'ai publié une traduction de Shear Yisrael sous le titre Why Antisemitism?, Jason Aronson, 1996. 
sérieux et pour tenter de réaliser tout ce qui est possible pour que le ciel et la terre puissent se rencontrer plus souvent dans les moments de grâce qui surgissent dans nos vies ; il croit que même s'il ne se sent pas apte à la tâche, il ne peut s'en soustraire ; et il croit que Dieu a confiance en sa valeur pour la réaliser.

\section{RÉSUMÉ}

La catégorie de l'humilité théologique met en évidence la nature infinie de l'Etre mystérieux de Dieu, la nature finie de notre compréhension et, par conséquent, la possibilité d'une pluralité d'approches légitimes du divin. Divers groupes religieux aperçoivent le divin grâce au sens cohérent qu'ils donnent aux événements et aux traditions dont ils héritent. L'humilité théologique, cependant, oblige les croyants à se rappeler qu'il s'agit de leur vérité, et qu'elle constitue par définition seulement un aperçu partiel de l'infini, suffisant néanmoins pour façonner et engager toute la vie d'une communauté. Ce point de vue peut fonder un pluralisme qui nous permet de tenir en même temps notre croyance en la vérité de notre propre tradition et l'affirmation de la vérité de la foi des autres.

\section{ABSTRACT}

The category of theological humility focuses on the infinite nature of God's mysterious Being, the finite nature of our understanding and, consequently, the possibility of a plurality of legitimate approaches to the divine. Various religious groups get a glimpse of the divine through the coherent meaning they give to the events and traditions to which they are heirs. Theological humility, however, requires believers to remember that this is their truth, of necessity of a partial glimpse of the infinite, sufficient nevertheless to shape and engage the total life of community. This position can be the basis of pluralism without relatitvism which enables us to hold on to both our belief in the truth of our own particular faith and the affirmation at the truth of other faiths. 